\title{
Improving Health and Reducing Comorbidity Associated with HIV: The Development of TAVIE en santé, a Web-Based Tailored Intervention to Support the Adoption of Health Promoting Behaviors among People Living with HIV
}

\author{
José Côté, ${ }^{1,2,3}$ Sylvie Cossette, ${ }^{3,4}$ Pilar Ramirez-Garcia, ${ }^{1,3}$ Geneviève Rouleau, ${ }^{1,2}$ \\ Patricia Auger, ${ }^{1,2}$ François Boudreau, ${ }^{5}$ and Marie-Pierre Gagnon ${ }^{6,7}$ \\ ${ }^{1}$ Research Centre of the Centre Hospitalier de l'Université de Montréal, 900 Saint Denis Street, Montréal, QC, Canada H2X 0A9 \\ ${ }^{2}$ Research Chair in Innovative Nursing Practices, 900 Saint Denis Street, Montréal, QC, Canada H2X 0A9 \\ ${ }^{3}$ Faculty of Nursing, Université de Montréal, 2375 Chemin de la Côte-Ste-Catherine, Montréal, QC, Canada H3T 1A8 \\ ${ }^{4}$ Research Center of the Montréal Heart Institute, 5000 Bélanger Street, Montréal, QC, Canada H1T 1C8 \\ ${ }^{5}$ Faculty of Nursing, Université du Québec à Trois-Rivières, 3351 Boul. des Forges, CP 500, Trois-Rivières, QC, Canada G9A 5H7 \\ ${ }^{6}$ Research Centre of the Centre Hospitalier Universitaire de Québec, 2705 Boulevard Laurier, Québec City, QC, Canada G1V 4 G2 \\ ${ }^{7}$ Faculty of Nursing Sciences, Université Laval, 1050 Avenue de la Médecine Local 3645, Québec City, QC, Canada G1V $0 A 6$
}

Correspondence should be addressed to José Côté; jose.cote@umontreal.ca

Received 22 September 2016; Accepted 30 January 2017; Published 14 March 2017

Academic Editor: Esteban Martinez

Copyright (C) 2017 José Côté et al. This is an open access article distributed under the Creative Commons Attribution License, which permits unrestricted use, distribution, and reproduction in any medium, provided the original work is properly cited.

\begin{abstract}
Background. In the domain of health behavior change, the deployment and utilization of information and communications technologies as a way to deliver interventions appear to be promising. This article describes the development of a web-based tailored intervention, TAVIE en santé, to support people living with HIV in the adoption of healthy behaviors. Methods. This intervention was developed through an Intervention Mapping (IM) framework and is based on the theory of planned behavior. Results. Crucial steps of IM are the selection of key determinants of behavior and the selection of useful theory-based intervention methods to change the targeted determinants (active ingredients). The content and the sequence of the intervention are then created based on these parameters. TAVIE en santé is composed of 7 interactive web sessions hosted by a virtual nurse. It aims to develop and strengthen skills required for behavior change. Based on an algorithm using individual cognitive data (attitude, perceived behavioral control, and intention), the number of sessions, theory-based intervention methods, and messages contents are tailored to each user. Conclusion. TAVIE en santé is currently being evaluated. The use of IM allows developing intervention with a systematic approach based on theory, empirical evidence, and clinical and experiential knowledge.
\end{abstract}

\section{Introduction}

The life expectancy of HIV-infected individuals with access to antiretroviral therapy (ART) is now measured in decades and, among those optimally treated, it can approach that of uninfected populations $[1,2]$. However, the long-term use of ART, the normal aging process, and the presence of certain lifestyle risk factors have been associated with onset of metabolic disorders, particularly glucose metabolism dysfunction and dyslipidemia [3-7]. These disorders, as well as other factors, can increase risk for diabetes and cardiovascular disease [8].

The interventions developed in recent years to prevent comorbidity among people living with HIV (PLHIV) have mostly targeted three lifestyle risk factors, namely, tobacco use $[9,10]$, physical inactivity $[11,12]$, and poor diet [13]. The literature in this regard is fragmentary. However, two recent literature reviews $[9,10]$ that examined, respectively, 9 and 10 studies of smoking cessation interventions for HIVinfected adults concluded that such interventions could be 
effective but that innovative individually tailored interventions needed to be developed for this population. Clearly, case management of PLHIV today must include risk-reducing interventions aimed at supporting PLHIV in their efforts to adopt positive health behaviors, especially quitting smoking, exercising, and developing better eating habits [14].

In the field of health behavior change interventions, the use of information and communications technologies (ICT) as a means of intervention delivery shows tremendous promise and the indubitable potential to promote healthy behaviors $[15,16]$. In a systematic review, Hou and colleagues (2014) concluded that web-based interventions produced positive results in terms of behavioral health outcomes [16]. In a meta-analysis of 40 randomized controlled trials (RCT) of web-delivered tailored health behavior change interventions, Lustria and colleagues (2013) reported that such interventions had a significantly greater effect on health behaviors, such as smoking cessation and healthy eating, compared with control conditions (e.g., nontailored intervention or waitlist control group) [15]. A tailored intervention can be defined as a change strategy devised for a specific person based on the person's individual characteristics identified beforehand through an individual evaluation [17-19]. The specificity of tailored interventions allows achieving greater significant results in terms of behavior change, especially if the person receives feedback adapted to the personal characteristics of their health behavior [20-22]. In this regard, the use of ICT seems, in theory, to offer many advantages in a public health perspective [23]. Uppermost among these is the capacity to disseminate a large amount of information formulated for individual needs, in a dynamic and sequential fashion, anywhere, anytime. Much of the user appeal of this approach derives from the possibility it affords of accessing customized information at one's convenience.

In light of the above, we developed a web-based tailored nursing intervention called TAVIE en santé to help PLHIV adopt healthy behaviors. The groundwork for the intervention was provided by the earlier creation of a virtual nursing intervention concept called TAVIE, the French acronym for Traitement Assistance Virtuelle Infirmière et Enseignement (Treatment Virtual Nurse Assistance and Teaching), and of an innovative web platform. The purpose of this paper is to describe how the TAVIE en santé intervention was developed.

\section{Methodology}

The web-based tailored nursing intervention was developed using the Intervention Mapping (IM) protocol developed by Bartholomew and colleagues [24, 25]. The strength of the protocol lies in the fact that it integrates theory and empirical evidence to develop an action plan for the primary, secondary, and tertiary levels of intervention. IM is a sixstep process. The first step consists in conducting a needs assessment to identify the health problem in the target population and associated determinants. In step two, matrices of change objectives are created to establish the behaviors and their determinants that should be targeted. Step three serves to select the theory-based intervention methods and the practical applications for changing the health behaviors via their determinants. Step four consists in developing the content and sequence of the intervention. Step five involves implementing the intervention and step six involves evaluating it.

Section 3 describes how the IM process was applied in developing the TAVIE en santé web-based tailored nursing intervention.

\section{Results}

3.1. Step 1: Needs Assessment. A needs assessment was carried out to document the lifestyle risk factors of PLHIV. In this case, determinants associated with the behavior changes sought were identified through screening of relevant literature.

3.1.1. Health Problem: Lifestyle Risk Factor. The selection of the topics of interest was based on clinical relevance and published data. Tobacco smoking has been found to be two to three times as prevalent among PLHIV as in the general population $[9,10,26-29]$, which means that $40 \%$ to $70 \%$ of PLHIV are smokers. Moreover, a literature review by SantosLozano and Garatachea (2011) suggested that PLHIV were drastically less active than the healthy population (e.g., as measured by number of steps walked per day and time spent doing physical activity) [30]. Also, a systematic review by Schuelter-Trevisol et al. (2012) revealed that up to $73 \%$ of PLHIV were sedentary [31]. Finally, research has shown that PLHIV were more likely to exceed intake recommendations for saturated fat $[32,33]$ and dietary cholesterol [34].

\subsubsection{Predictors of Healthy Behaviors: Theory of Planned} Behavior. The meta-analyses carried out by Armitage and Conner (2001), Godin and Kok (1996), Sheeran (2002), and Webb and Sheeran (2006) suggested the Ajzen's theory of planned behavior was very good for predicting and explaining numerous health behaviors [35-39]. According to this theory, whether a health behavior is adopted is determined by intention (motivation), which in turn is explained by attitude, subjective norm, and perceived behavioral control. Attitude and subjective norm influence intention, which predicts whether the behavior will be adopted. However, perceived behavioral control is a determinant that can influence the behavior directly when the behavior is not under volitional control. Within the framework of the proposed intervention aimed at quitting smoking, engaging in physical activity, or adopting a healthy diet, three determinants of behavior were targeted: intention, attitude, and perceived behavioral control (see Figure 1).

3.2. Step 2: Matrix of Change Objectives. The matrix of change objectives is the basic tool used in IM for conceptualizing and developing interventions [24]. The matrix is created by the intersection of performance objectives and determinants (i.e., factors that explain and/or predict the adoption of healthy behaviors). The endpoints, or proximal program objectives, state what must be learned and performed by the target group in order to optimize health behaviors. 


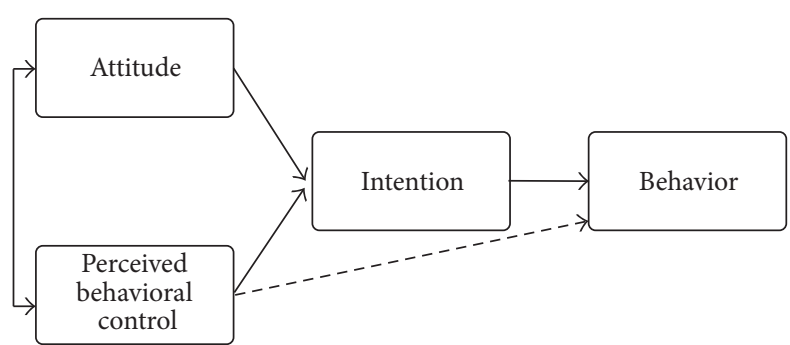

Figure 1: Targeted determinants of behavior in the TAVIE en santé intervention (figure adapted from Ajzen (1991) in Godin (2012) [35]).

The first task in step 2 is to set expected intervention outcomes. The aim of our nursing intervention is the adoption of the following healthy behaviors: smoking cessation, physical activity, or healthy eating. Intervention recipients are asked to choose which of these three behaviors they wish to change. The second task in step 2 is to subdivide behavioral outcomes into performance objectives. These describe what people need to do in order to achieve the desired change: (1) identify the health behaviors to adopt; (2) choose to adopt one of the proposed behaviors and undertake the process of adopting the behavior, begin the behavior, and act accordingly; and (3) engage in and consolidate the behavior, overcome possible barriers by deploying means and reminding oneself of the benefits of adopting the behavior. The third task in step 2 is to select and refine the key determinants of behavior amenable to change. Table 1 presents the matrix of change objectives.

\subsection{Step 3: Theory-Based Intervention Methods and Practical} Applications. Step 3 in IM consists in selecting useful theorybased intervention methods to change the targeted determinants of behaviors. Practical applications, for their part, are specific techniques or strategies that serve to organize and operationalize the theoretical methods. In our case, three methods are used for all the targeted determinants: tailoring, modeling, and feedback/reinforcement. Specific methods are also used for each determinant. To change attitude, the following methods are employed: behavioral belief selection and persuasive communication. To identify behavioral beliefs, the virtual nurse in the intervention provides information on the benefits of the target behavior. She invites participants to identify the advantages and disadvantages for them of adopting the chosen behavior. She also refers to examples provided by other PLHIV to help with the process. Here is an example of what the virtual nurse might say:

Right now, you have little or no intention of engaging in physical activity and barely see any advantage to doing this. Tell yourself that you are not the only one in this situation! Taking steps to start doing more physical activity is no simple task. Many people living with HIV find advantages in physical activity. Being active makes them feel fit, helps them control their weight, and improves flexibility in order to prevent aches, pains and muscular atrophy. It also improves oxygen flow to the brain. Studies have shown that adopting habits such as performing physical activity reduces the risk of heart disease and diabetes. Additionally, it helps unwind and restore your sense of well-being. I invite you to listen to Daniel's story about the advantages and disadvantages of physical activity.

To improve perceived behavioral control, the following methods are deployed: control belief selection, coping planning, and verbal persuasion. Finally, there are two preferred methods for acting on intention: implementation of intention and goal setting. Table 2 gives the targeted determinants and their corresponding theory-based intervention methods, definitions, parameters for use, and applications/messages. The table is modeled on the one presented in Bartholomew et al. (2011) [24].

3.4. Step 4: Content and Sequences of the Intervention. TAVIE en santé is a tricomponent web-based tailored intervention facilitated by a virtual nurse. It is meant to address smoking cessation (SC), physical activity (PA), and healthy eating (HE). The virtual nurse is at the heart of each web session. This virtual nurse is in fact a real nurse who acts as a "virtual coach" and interacts with PLHIV asynchronously via the medium of video clips. The role of this virtual nurse is to provide education and to propose strategies to participants to support them in adopting their chosen health behavior. She also provides positive reinforcement and feedback on the significant elements of the previous session. This ensures follow-up prior to each new session by verifying the participant understanding of proposed skills and their application in daily life. Aside from delivering tailored teaching, the virtual nurse also refers to the experiences of other people who have been able to cope with similar situations successfully.

Each component (SC, PA, and HE) includes seven interactive web sessions lasting 5 to 10 minutes. The average duration of each component is 50 minutes. Each web session has a distinct objective. The first session focuses on identifying the advantages and disadvantages of adopting the chosen behavior. The second session aims to help the participant remember the advantages of adopting this behavior. The third and fourth sessions are geared to identifying difficult situations and barriers as well as ways to overcome them. The fifth session is intended to help the participant formulate an action plan to facilitate adopting the behavior, and the sixth session is supposed to reinforce this action plan. The seventh session serves as a booster to consolidate what the participants learned during the intervention.

The intervention is tailored on level of intention, attitude, and perceived control. Participants are assigned one of three profiles generated by a computer algorithm (see Figure 2). Profile 1 (P1) is marked by low attitude regarding adopting the chosen behavior and requires completing all seven sessions of the intervention. Profile 2 (P2) is characterized by low perceived control and requires completing five sessions (sessions 3 to 7). Profile 3 (P3) corresponds to high intention to change the chosen behavior and requires completing only three sessions (5 to 7). It is assumed that a person with high intention to change (P3) will require less virtual support than a person with low intention to change (P1) or with 
TABLE 1: Matrix of objectives.

Performance objectives

Intention and behavior determinants and change objectives

Attitude

Perceived control

Intention

(PO1) Identify/choose a health behavior to adopt.

(PO2) Make the decision to adopt a proposed behavior. Engage in a behavior adoption process.

Begin adopting the behavior and act accordingly.

(PO3) Act and consolidate the behavior, overcome potential barriers by deploying means and reminding the advantages of adopting the behavior.
(A1) Understand the importance of adopting the health behavior.

(A2) Identify the advantages and disadvantages of adopting the chosen behavior.

Identify the positive and negative

emotions in adopting the chosen behavior.

(A3) Evaluate adoption of the behavior positively and focus on the advantages of adopting the chosen behavior.
(PC1) Assess the capacity to adopt the health behavior.

(PC2) Identify the barriers and factors that may hinder or facilitate adoption of the chosen behavior.

Identify ways of overcoming the barriers and negative emotions and the external resources that may help adopt the chosen behavior.

(PC3) Believe in the ability to use the factors and external resources that may facilitate adoption of the behavior.

Use these problem-solving strategies.
(I1) Formulate an intention to motivate the adoption of the behavior.

(I2) Formulate an action plan describing when, where, and how to adopt the chosen behavior.

Anticipate/identify/recognize the barriers and find ways to overcome and foresee the difficulties.

(I3) Carry out/reinforce the action plan and act on the barriers encountered. low perceived control over change (P2). Each profile receives specific, theory-based intervention methods and tailored messages. For example, belief selection and persuasive communication are used to change a participant's attitude towards their chosen behavior. Coping, planning, and role modeling are used to improve perceived behavioral control and goal setting, whereas implementation of intention is used to act on intention.

For the purpose of developing the intervention content (i.e., messages, case stories, scenarios reflecting the reality, and lived experience of PLHIV), a focus group was held with six PLHIV ( 3 men and 3 women), 30 to 70 years old. The group served to identify behavioral and control beliefs inherent to each behavior. Messages were created in line with focus group content, the theoretical methods used, and the determinants of behaviors chosen.

After completing the web sessions corresponding to their profile, participants can revisit all previous sessions as often as they wish via a table of contents. The fact that TAVIE en santé is available in both French and English makes it possible to reach a wider audience. The multimedia content of the intervention consists of nearly 350 web pages with 296 videos, 164 animated narrations, and 84 PDF files containing information and tools (see Figure 3 ).

Our unique team brings together researchers from a variety of disciplines (nursing, medicine, social work, health promotion, and computer science) to pool their expertise in AIDS, cardiovascular disease, and diabetes for a common cause: the development of innovative interventions for the treatment of health problems experienced by PLHIV. Moreover, the content of the intervention has been validated by different health professionals, including a nutritionist, a kinesiologist, nurses, and physical activity research experts. RCT with parallel groups is currently ongoing across Canada.
To participate in the study, PLHIV must be $\geq 18$ years old, able to read/understand French or English, and have Internet access. A convenience sample of 750 participants will be assigned randomly either to an experimental group exposed to the TAVIE en santé intervention or to a control group with access to informative websites (1:1 allocation ratio). Various health behaviors and cognition and health indicators will be assessed at preintervention (T0), 3-month follow-up (T3), and 6-month follow-up (T6). The procedure for this online study will be based on our earlier HIV-Medic Online study [41]. The trial protocol for evaluating the TAVIE en santé intervention has been published elsewhere [42].

\section{Discussion}

The purpose of this article was to present results obtained at each step in the development of the TAVIE en santé intervention for PLHIV using the IM process and to contribute to the scientific literature on web-based tailored interventions.

There are well-established conceptual frameworks to guide the development of health interventions [24, 43]. The IM method that we used is a systematic approach that draws on theory, empirical evidence, and clinical and experiential knowledge. Identifying the health problem and determinants to target and selecting the most appropriate theoretical methods and practical applications to address these determinants are key steps in the process. Choosing theory-based intervention methods and practical applications suited to the health-related behavior to change and the determinants to target is crucial as well. Michie and colleagues (2008, 2011) have pleaded for the use of theory-based approaches not only to understand behaviors but also to guide intervention design $[44,45]$. Unfortunately, interventions are more often theoryinspired than they are theory-based.

For the development of TAVIE en santé, IM was chosen over and above other methodologies considering its 


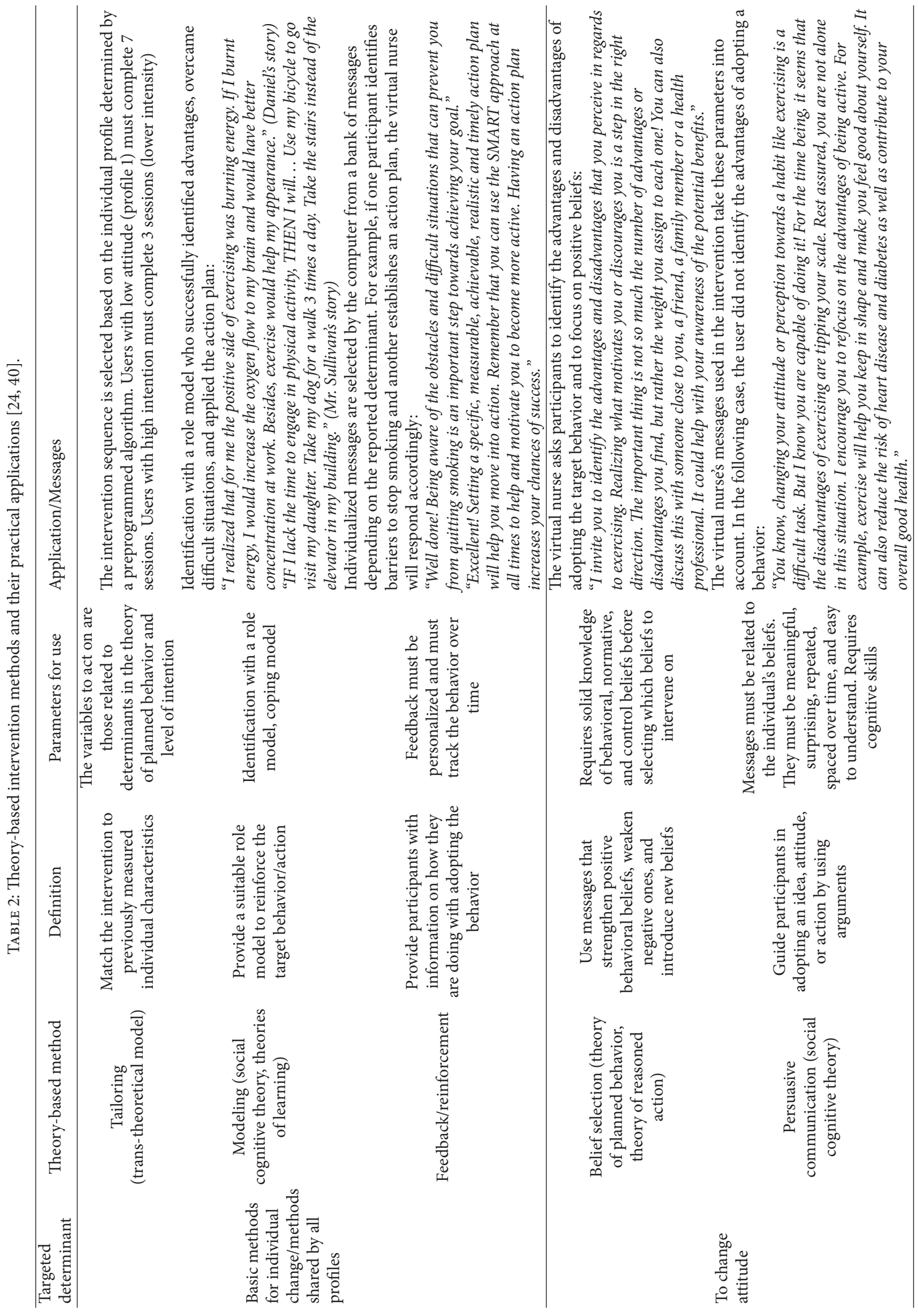




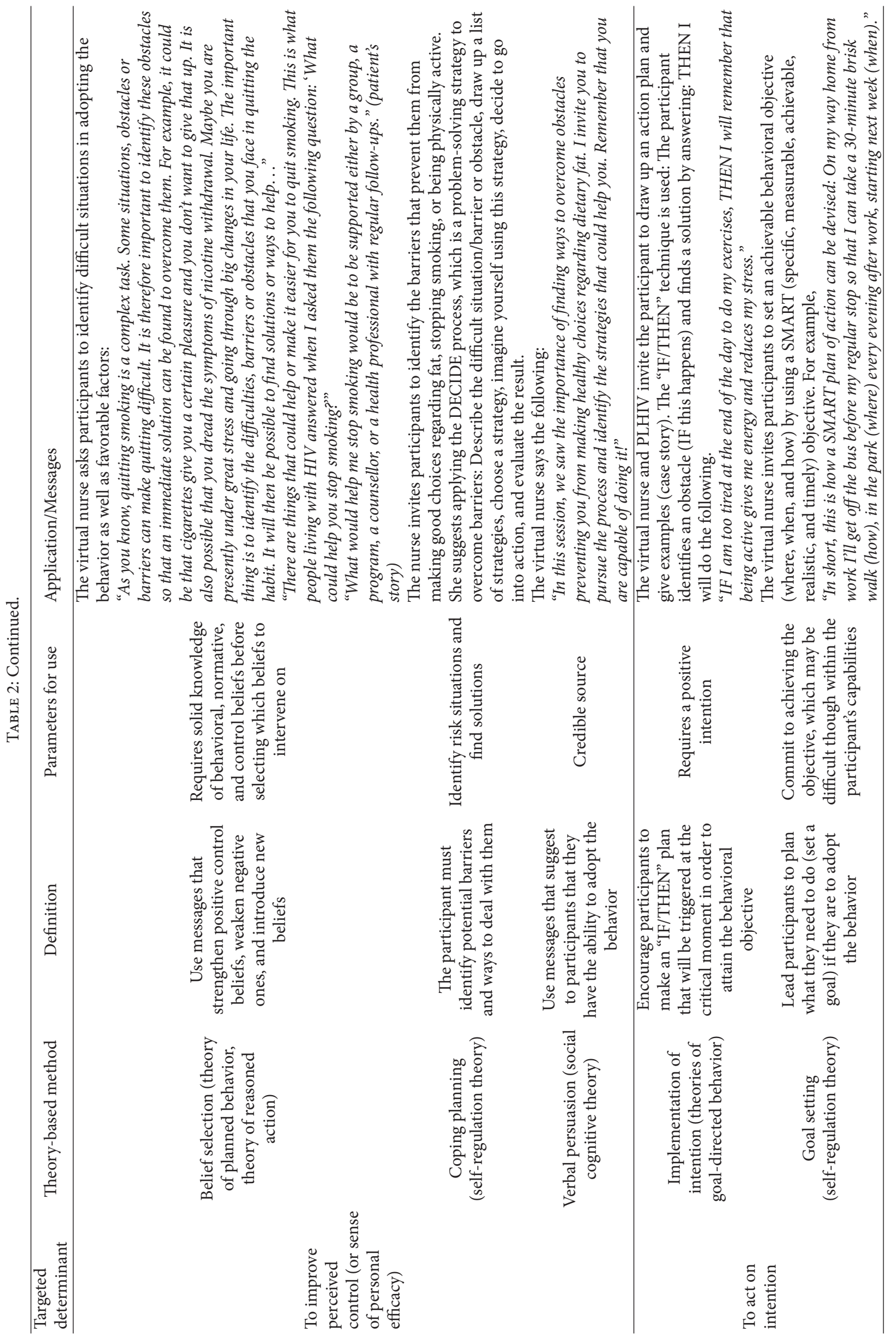




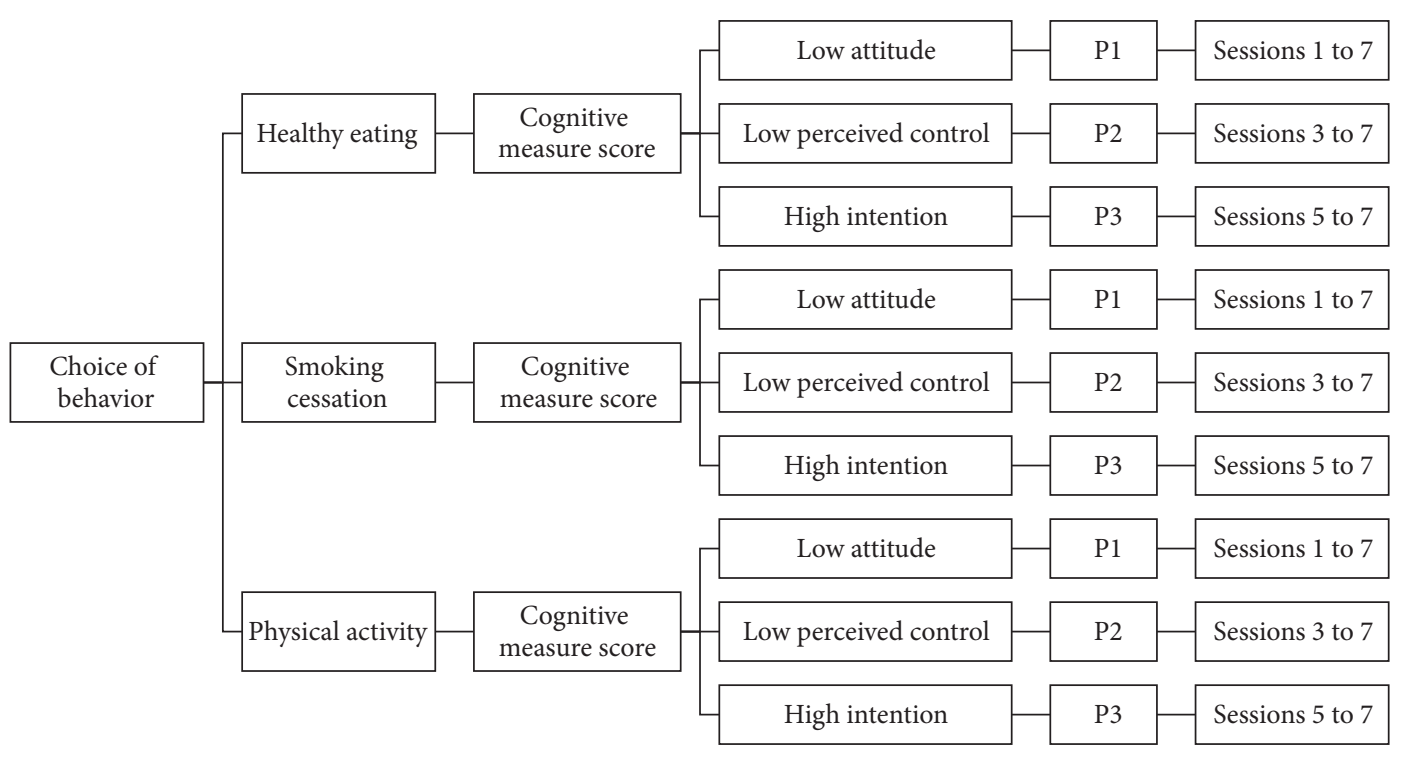

Figure 2: Profile attribution.
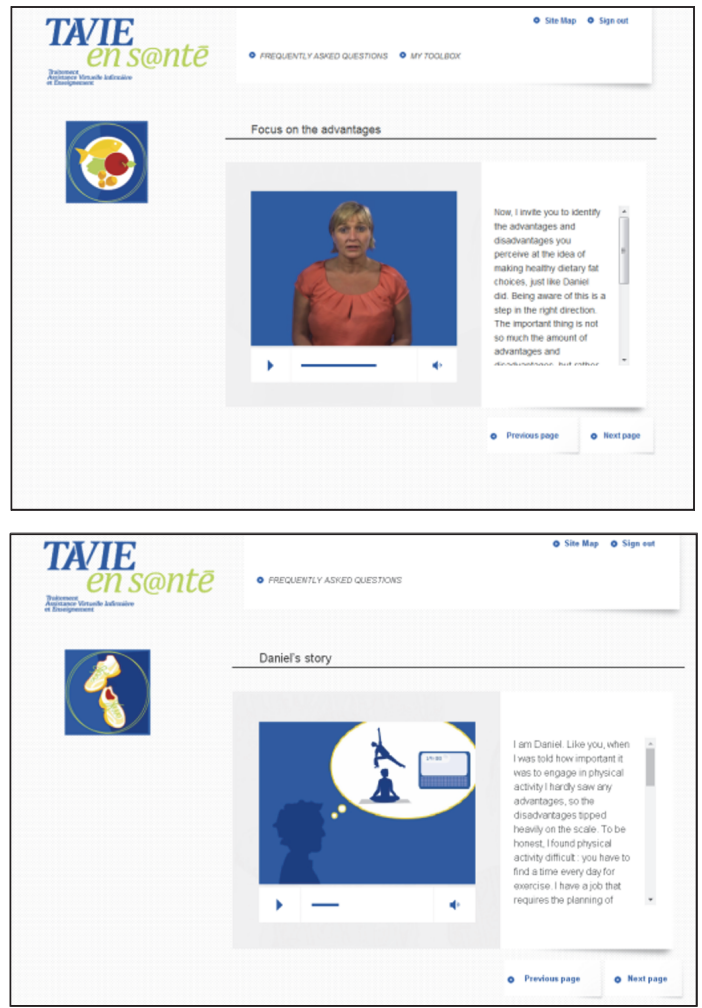

Figure 3: Screenshots of a TAVIE en santé web page.

strengths, added value, and the expertise of the research team associated with this approach. During the development process, some steps were more challenging than others. Steps 1 and 2 were useful in targeting the problem and its determinants. They provided answers to important questions that were prerequisites to the development of the intervention such as the following: what is the goal of the intervention?
What are we trying to change? This problem came to light due to a clinical preoccupation to prevent and manage comorbidities among people living with HIV. Three health behaviors were selected: tobacco use, physical inactivity, and poor diet. The current state of empirical and theoretical knowledge facilitated the identification of healthy behavior predictors which became the targeted determinants, namely, intention, attitude, and perceived behavioral control. The challenges arose mainly during steps 3 and 4 . We needed to operationalize the content and to find answers to questions such as the following: what strategies for change are available? What and how strategies should be used? The operationalization of the theoretical methods in practical applications and the content development were particularly challenging. During these steps, clinical and experiential knowledge from professionals and patients were highly relevant: they greatly contributed to defining the specific contents and sequences of the intervention. Developing the clinical contents of TAVIE en santé required multiple rounds of validation and revisions throughout the process.

While IM allows detailing each step and component of an intervention, it offers little guidance regarding webbased content delivery [46]. In this regard, Morrison and colleagues (2012) stated that existing intervention development approaches needed to be supplemented by frameworks to guide the delivery of content via ICT [47]. Applying critical interpretive synthesis, Morrison and colleagues (2012) proposed four design features that might mediate the effect of ICT interventions on outcomes: self-management, contacts with intervention, tailoring, and social context and support [47]. Our web-based tailored nursing intervention is meant to empower individuals to adopt healthy behaviors. Through asynchronous contacts, the virtual nurse that facilitates the intervention provides tailored coaching and support to this end. The intervention also presents a role model: a virtual patient in a situation similar to the user's, who shares their experience of adopting the proposed skills and strategies. This 
is the virtual environment that was created to provide a social context and social support.

Health behavior change constitutes a real challenge. In a systematic review of the literature on communication-related behavior change techniques used in face-to-face lifestyle interventions in primary care, Noordman and colleagues (2012) found that 28 of the 50 studies assessed reported significantly favorable health outcomes [48]. But what of tailored interventions of the sort delivered online? Can a web-based intervention alone help change behaviors? In their systematic review of reviews on online prevention aimed at lifestyle behaviors, Kohl and colleagues (2013) found that overall effects of these interventions are positive but the effect sizes appear to be small [49]. In their meta-analysis of webdelivered tailored health behavior change interventions $(n=$ 40 RCT), Lustria and colleagues (2013) found that these were most successful when they targeted general populations $(n=$ $26)$ rather than chronically ill patients $(n=6$, individuals not screened for any particular chronic illness or other disease factors) [15]. Similarly, according to Murray (2012), webbased interventions for people living with chronic conditions remained a challenge and might be less successful [50].

Finally, people living with a chronic health condition often fail to perceive lifestyle behavior changes as an imperative, especially compared with the other health-related challenges they face, such as adherence to medication intake. Consequently, they often lack the readiness and engagement to enact these changes. The issue of engagement in webbased interventions, that is, the fact that not all participants follow or complete online interventions according to plan, is well documented in the literature [51-53]. In their systematic review, for example, Kelders and colleagues (2012) found that only about $50 \%$ of participants adhered rigorously to interventions of the sort [52]. Research has sought to identify the characteristics of eHealth intervention nonengagers [54, 55]. In their study, Kelders and colleagues (2011) found that older age and chronic condition predicted nonuse of a webbased intervention to promote healthy eating and exercising behavior [54]. In their systematic review, Kelders and colleagues (2012) proposed various strategies to boost engagement: shorter intervention segments, more dialogue-based support, and interaction with a counselor [52]. They recommended taking these elements into account when designing a web-based intervention. Alkhaldi and colleagues (2016), instead, found that engagement could be bolstered by technology-based strategies such as the use of emails, text messages, and telephone calls [51]. These findings must be interpreted with caution, however, as the field of engagement strategies is still only at a nascent stage.

\section{Conclusion}

In conclusion, the systematic use of IM allowed us to detail all of the steps and components of the intervention following recommendations specific to tailored interventions and the TIDieR checklist [56]. These instruments and guidelines make for better reporting of the content of behavior change interventions, which is essential to help develop new interventions, refine existing ones, and replicate and disseminate them on a larger scale. The use of IM allows avoiding "black box" interventions that, while they may prove effective, are difficult to repeat and explain for lack of implementation data and an understanding of underlying mechanisms.

\section{Disclosure}

Previous presentations of this paper included oral presentations at the 6e Congrès Mondial des Infirmières et Infirmiers Francophones, Montréal, Canada (June 2015), and poster presentation at the 25th Annual Canadian Conference on HIV/AIDS Research, Winnipeg, Canada (May 2016).

\section{Competing Interests}

The authors declare that there is no conflict of interests regarding the publication of this paper.

\section{Acknowledgments}

This study is funded by the Canadian Institutes of Health Research (CIHR, 2013-2016). José Côté has received a clinical research bursary (Senior) from the Fonds de Recherche $d u$ Québec-Santé (FRQS, 2013-2017) to support her research program on innovative virtual interventions that are intended for persons living with a chronic health problem. The TAVIE platform received financial support from the Réseau Sidami du FRSQ. The authors would like to especially thank the participants who participated in the focus group and the professionals who made the development of this intervention possible.

\section{References}

[1] L. F. Johnson, J. Mossong, R. E. Dorrington et al., "Life expectancies of South african adults starting antiretroviral treatment: collaborative analysis of cohort studies," PLoS Medicine, vol. 10, no. 4, Article ID e1001418, 2013.

[2] F. Nakagawa, M. May, and A. Phillips, "Life expectancy living with HIV: recent estimates and future implications," Current Opinion in Infectious Diseases, vol. 26, no. 1, pp. 17-25, 2013.

[3] N. Friis-Møller, R. Weber, P. Reiss et al., "Cardiovascular disease risk factors in HIV patients-association with antiretroviral therapy. Results from the DAD study," AIDS, vol. 17, no. 8, pp. 1179-1193, 2003.

[4] R. A. Bradbury and K. Samaras, "Antiretroviral therapy and the human immunodeficiency virus-improved survival but at what cost?" Diabetes, Obesity and Metabolism, vol. 10, no. 6, pp. 441-450, 2008.

[5] A. A. Butt, K. McGinnis, M. C. Rodriguez-Barradas et al., "HIV infection and the risk of diabetes mellitus," AIDS, vol. 23, no. 10, pp. 1227-1234, 2009.

[6] K. Samaras, "Prevalence and pathogenesis of diabetes mellitus in HIV-1 infection treated with combined antiretroviral therapy," Journal of Acquired Immune Deficiency Syndromes, vol. 50, no. 5, pp. 499-505, 2009.

[7] P. E. Sax, "Assessing risk for cardiovascular disease in patients with human immunodeficiency virus: why it matters," Circulation, vol. 121, pp. 620-622, 2010.

[8] J. H. Stein and P. Y. Hsue, "Inflammation, immune activation, and CVD risk in individuals with HIV infection," Journal of 
the American Medical Association, vol. 308, no. 4, pp. 405-406, 2012.

[9] P. A. Cioe, "Smoking cessation interventions in HIV-infected adults in north america: a literature review," Journal of Addictive Behaviors Therapy \& Rehabilitation, vol. 2, no. 3, Article ID 1000112, 2013.

[10] G. Moscou-Jackson, Y. Commodore-Mensah, J. Farley, and M. DiGiacomo, "Smoking-cessation interventions in people living with HIV infection: a systematic review," Journal of the Association of Nurses in AIDS Care, vol. 25, no. 1, pp. 32-45, 2014.

[11] J. R. Jaggers, W. Dudgeon, S. N. Blair et al., "A home-based exercise intervention to increase physical activity among people living with HIV: study design of a randomized clinical trial," BMC Public Health, vol. 13, no. 1, article no. 502, 2013.

[12] R. Roos, H. Myezwa, H. Van Aswegen, and E. Musenge, "Effects of an education and home-based pedometer walking program on ischemic heart disease risk factors in people infected with HIV: a randomized trial," Journal of Acquired Immune Deficiency Syndromes, vol. 67, no. 3, pp. 268-276, 2014.

[13] R. K. Lazzaretti, R. Kuhmmer, E. Sprinz, C. A. Polanczyk, and J. P. Ribeiro, "Dietary intervention prevents dyslipidemia associated with highly active antiretroviral therapy in human immunodeficiency virus type 1-infected individuals: a randomized trial," Journal of the American College of Cardiology, vol. 59, no. 11, pp. 979-988, 2012.

[14] K. Samaras, "The burden of diabetes and hyperlipidemia in treated HIV infection and approaches for cardiometabolic care," Current HIV/AIDS Reports, vol. 9, no. 3, pp. 206-217, 2012.

[15] M. L. A. Lustria, S. M. Noar, J. Cortese, S. K. Van Stee, R. L. Glueckauf, and J. Lee, "A meta-analysis of web-delivered tailored health behavior change interventions," Journal of Health Communication, vol. 18, no. 9, pp. 1039-1069, 2013.

[16] S. Hou, S. R. Charlery, and K. Roberson, "Effects of an education and home-based pedometer walking program on ischemic heart disease risk factors in people infected with HIV: a randomized trial," Health Psychology and Behavioral Medicine, vol. 2, no. 1, pp. 455-481, 2014.

[17] M. W. Kreuter, E. M. Clark, D. L. Oswald, and F. C. Bull, "Understanding how people process health information: a comparison of tailored and nontailored weight-loss materials," Health Psychology, vol. 18, no. 5, pp. 487-494, 1999.

[18] M. W. Kreuter, D. Farrell, L. Olevitch, and L. Brennan, Tailored Health Messages: Customizing Communication with Computer Technology, Lawrence Erlbaum, Mahwah, NJ, USA, 1999.

[19] M. W. Kreuter and C. S. Skinner, “Tailoring: what's in a name?" Health Education Research, vol. 15, no. 1, pp. 1-4, 2000.

[20] A. Dijkstra, "Working mechanisms of computer-tailored health education: evidence from smoking cessation," Health Education Research, vol. 20, no. 5, pp. 527-539, 2005.

[21] A. Dijkstra and H. De Vries, "The development of computergenerated tailored interventions," Patient Education and Counseling, vol. 36, no. 2, pp. 193-203, 1999.

[22] H. De Vries and J. Brug, "Computer-tailored interventions motivating people to adopt health promoting behaviours: introduction to a new approach," Patient Education and Counseling, vol. 36, no. 2, pp. 99-105, 1999.

[23] B. Oldenburg, C. B. Taylor, A. O’Neil, F. Cocker, and L. D. Cameron, "Using new technologies to improve the prevention and management of chronic conditions in populations," Annual Review of Public Health, vol. 36, pp. 483-505, 2015.
[24] L. Bartholomew, G. S. Parcel, G. Kok, N. H. Gottlieb, and M. E. Fernández, Planning Health Promotion Programs: An Intervention Mapping Approach, Wiley, New York, NY, USA, 3th edition, 2011.

[25] L. K. Bartholomew, G. S. Parcel, G. Kok, and N. H. Gottlieb, Planning Health Promotion Programs: An Intervention Mapping Approach, Jossey-Bass, 2006.

[26] A. R. Lifson and H. A. Lando, "Smoking and HIV: prevalence, health risks, and cessation strategies," Current HIV/AIDS Reports, vol. 9, no. 3, pp. 223-230, 2012.

[27] R. Mdodo, E. L. Frazier, S. R. Dube et al., "Cigarette smoking prevalence among adults with HIV compared with the general adult population in the United States: cross-sectional surveys," Annals of Internal Medicine, vol. 162, no. 5, pp. 335-344, 2015.

[28] S. Nahvi and N. A. Cooperman, "Review: the need for smoking cessation among HIV-positive smokers," AIDS Education and Prevention, vol. 21, supplement 3, pp. 14-27, 2009.

[29] J. M. Tesoriero, S. M. Gieryic, A. Carrascal, and H. E. Lavigne, "Smoking among HIV positive New Yorkers: prevalence, frequency, and opportunities for cessation," AIDS and Behavior, vol. 14, no. 4, pp. 824-835, 2010.

[30] A. Santos-Lozano and N. Garatachea, "Physical activity measurements using accelerometers and pedometers in HIVinfected people," Journal of AIDS and Clinical Research, vol. 2, no. 5, article no. 126, 2011.

[31] F. Schuelter-Trevisol, F. H. Wolff, P. R. Alencastro et al., "Physical activity: do patients infected with HIV practice? How much? A systematic review," Current HIV Research, vol. 10, no. 6, pp. 487497, 2012.

[32] K. Klassen and L. M. Goff, "Dietary intakes of HIV-infected adults in urban UK," European Journal of Clinical Nutrition, vol. 67, no. 8, pp. 890-893, 2013.

[33] K. V. Giudici, A. C. F. L. Duran, and P. C. Jaime, "Inadequate food intake among adults living with HIV," São Paulo Medical Journal, vol. 131, no. 3, pp. 145-152, 2013.

[34] T. Joy, H. M. Keogh, C. Hadigan et al., "Dietary fat intake and relationship to serum lipid levels in HIV-infected patients with metabolic abnormalities in the HAART era," AIDS, vol. 21, no. 12, pp. 1591-1600, 2007.

[35] G. Godin, Les Comportements dans le Domaine de la Santé: Comprendre Pour Mieux Intervenir, Les Presses de l'Université de Montréal, 2012.

[36] C. J. Armitage and M. Conner, "Efficacy of the theory of planned behaviour: a meta-analytic review," British Journal of Social Psychology, vol. 40, no. 4, pp. 471-499, 2001.

[37] G. Godin and G. Kok, "The theory of planned behavior: a review of its applications to health- related behaviors," American Journal of Health Promotion, vol. 11, no. 2, pp. 87-98, 1996.

[38] P. Sheeran, "Intention-behavior relations: a conceptual and empirical review," European Review of Social Psychology, vol. 12, no. 1, pp. 1-36, 2002.

[39] T. L. Webb and P. Sheeran, "Does changing behavioral intentions engender behavior change? A meta-analysis of the experimental evidence," Psychological Bulletin, vol. 132, no. 2, pp. 249268, 2006.

[40] H. Gagnon, J. Côté, S. Tessier, and N. April, “Développement d'une plateforme Web pour réduire l'usage de cannabis chez les jeunes qui fréquentent les centres d'éducation des adultes," Drogues, santé et société, vol. 11, no. 2, pp. 1-17, 2012.

[41] J. Côté, G. Godin, Y.-G. Guéhéneuc et al., "Evaluation of a realtime virtual intervention to empower persons living with HIV 
to use therapy self-management: study protocol for an online randomized controlled trial," Trials, vol. 13, article no. 187, 2012.

[42] J. Côté, S. Cossette, P. Ramirez-Garcia et al., "Evaluation of a Web-based tailored intervention (TAVIE en santé) to support people living with HIV in the adoption of health promoting behaviours: an online randomized controlled trial protocol," BMC Public Health, vol. 15, no. 1, article 1042, 2015.

[43] L. Green and M. Kreuter, Health and Promotion Planning: An Educational and Ecological Approach, Mayfield, Mountain View, Calif, USA, 1999.

[44] S. Michie, M. M. van Stralen, and R. West, "The behaviour change wheel: a new method for characterising and designing behaviour change interventions," Implementation Science, vol. 6, no. 1, article 42, 2011.

[45] S. Michie, M. Johnston, J. Francis, W. Hardeman, and M. Eccles, "From theory to intervention: mapping theoretically derived behavioural determinants to behaviour change techniques," Applied Psychology, vol. 57, no. 4, pp. 660-680, 2008.

[46] C. A. Pellegrini, J. Steglitz, and S. A. Hoffman, "e-Health intervention development: a synopsis and comment on 'What design features are used in effective e-Health interventions? A review using techniques from critical interpretive synthesis," Translational Behavioral Medicine, vol. 4, no. 4, pp. 342-345, 2014.

[47] L. G. Morrison, L. Yardley, J. Powell, and S. Michie, "What design features are used in effective e-health interventions? A review using techniques from Critical Interpretive Synthesis," Telemedicine Journal and E-health, vol. 18, no. 2, pp. 137-144, 2012.

[48] J. Noordman, T. van der Weijden, and S. van Dulmen, "Communication-related behavior change techniques used in face-toface lifestyle interventions in primary care: a systematic review of the literature," Patient Education and Counseling, vol. 89, no. 2, pp. 227-244, 2012.

[49] L. F. M. Kohl, R. Crutzen, and N. K. De Vries, "Online prevention aimed at lifestyle behaviors: a systematic review of reviews," Journal of Medical Internet Research, vol. 15, no. 7, article no. e146, 2013.

[50] E. Murray, "Web-based interventions for behavior change and self-management: potential, pitfalls, and progress," Journal of Medical Internet Research, vol. 1, no. 2, 2012.

[51] G. Alkhaldi, F. L. Hamilton, R. Lau, R. Webster, S. Michie, and E. Murray, "The Effectiveness of prompts to promote engagement with digital interventions: a systematic review," Journal of Medical Internet Research, vol. 18, no. 1, article e6, 2016.

[52] S. M. Kelders, R. N. Kok, H. C. Ossebaard, and J. E. W. C. van Gemert-Pijnen, "Persuasive system design does matter: a systematic review of adherence to web-based interventions," Journal of Medical Internet Research, vol. 14, no. 6, article e152, 2012.

[53] D. A. Reinwand, R. Crutzen, I. Elfeddali et al., "Impact of educational level on study attrition and evaluation of web-based computer-tailored interventions: results from seven randomized controlled trials," Journal of Medical Internet Research, vol. 17, no. 10, article e228, 2015.

[54] S. M. Kelders, J. E. W. C. Van Gemert-Pijnen, A. Werkman, N. Nijland, and E. R. Seydel, "Effectiveness of a web-based intervention aimed at healthy dietary and physical activity behavior: a randomized controlled trial about users and usage," Journal of Medical Internet Research, vol. 13, no. 2, article e32, 2011.

[55] D. N. Schulz, F. Schneider, H. de Vries, L. A. D. M. van Osch, P. W. M. van Nierop, and S. P. J. Kremers, "Program completion of a web-based tailored lifestyle intervention for adults: differences between a sequential and a simultaneous approach," Journal of Medical Internet Research, vol. 14, no. 2, article e26, 2012.

[56] T. C. Hoffmann, P. P. Glasziou, I. Boutron et al., "Better reporting of interventions: template for intervention description and replication (TIDieR) checklist and guide," BMJ, vol. 348, Article ID g1687, 2014. 


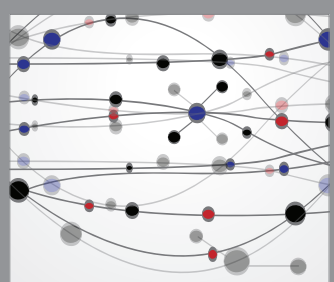

The Scientific World Journal
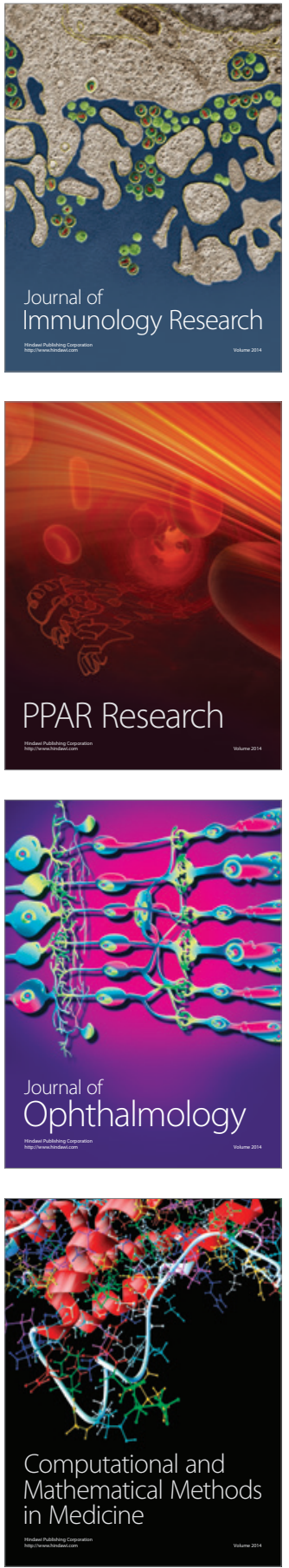

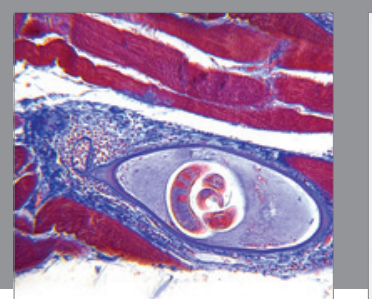

Gastroenterology Research and Practice
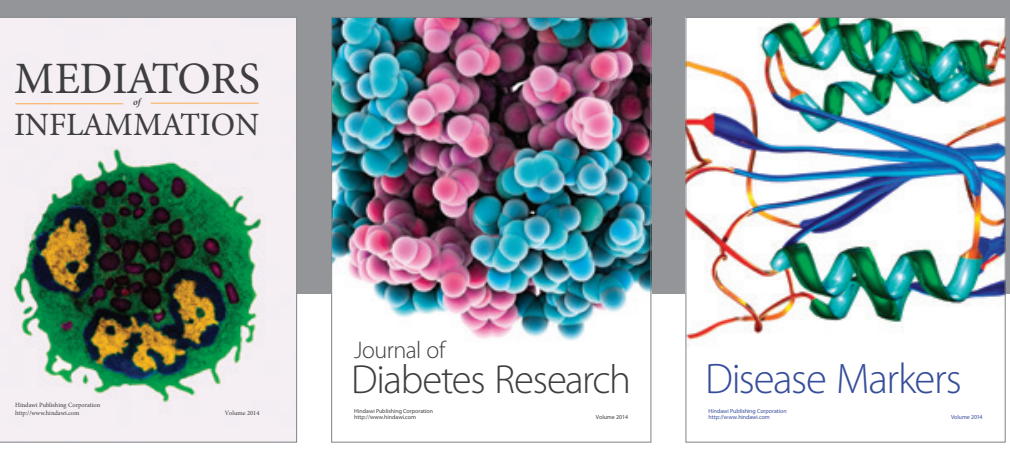

Disease Markers

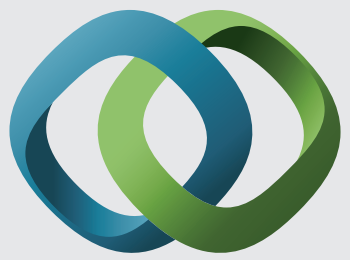

\section{Hindawi}

Submit your manuscripts at

https://www.hindawi.com
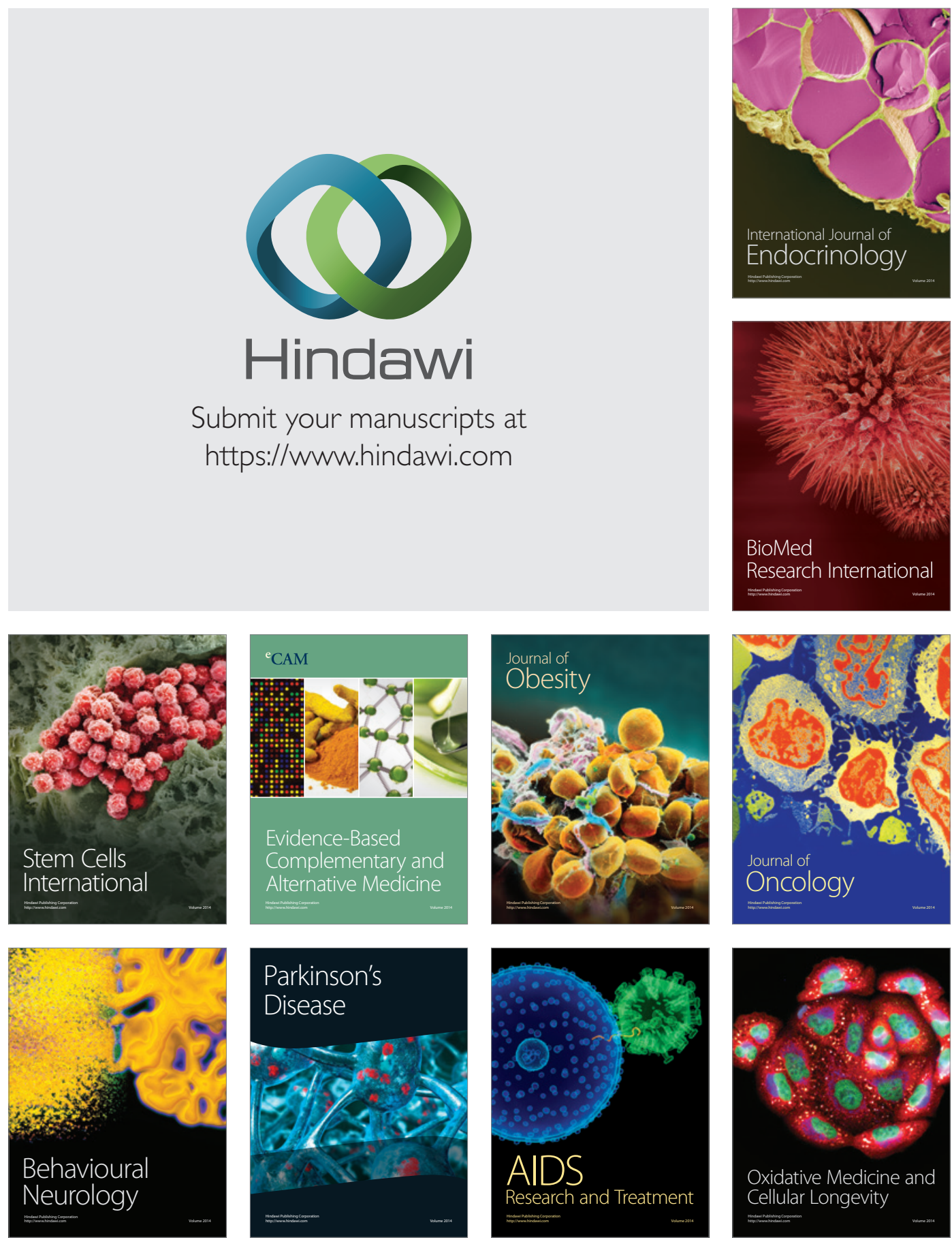